\title{
Choroidal Neovascularization Induced by Immunogenic Alteration of the Retinal Pigment Epithelium in Dengue Fever
}

\author{
Carlos Eduardo Veloso ${ }^{a} \quad$ Ursula Schmidt-Erfurth $^{\mathrm{b}}$ Márcio B. Nehemy ${ }^{\mathrm{a}}$ \\ ${ }^{a}$ Retina Section, Department of Ophthalmology, Federal University of Minas Gerais, Belo \\ Horizonte, Brazil; ${ }^{b}$ Department of Ophthalmology and Optometry, Medical University of \\ Vienna, Vienna, Austria
}

\section{Key Words}

Dengue maculopathy · Choroidal neovascularization · Complement activation ·

Antiangiogenic therapy

\begin{abstract}
Purpose: To report the first case of choroidal neovascularization (CNV) secondary to dengue fever. Case Report: A 54-year-old female was referred to our department with blurred vision and metamorphopsia in her left eye. Two weeks earlier, she had presented all of the classic symptoms of dengue fever including a positive serology. Her best-corrected visual acuity (BCVA) was 20/150 in the left eye. She underwent a fundus examination, fluorescein angiography (FA) and spectral domain optical coherence tomography. Results: All findings were consistent with CNV secondary to dengue fever. FA revealed a classic CNV associated with focal retinal pigment epithelium (RPE) destruction and detachment. Three consecutive monthly injections of intravitreal ranibizumab resulted in functional and anatomical improvement for as long as 6 months with a BCVA of 20/25. However, CNV recurred 2 years later, again with an improvement after ranibizumab therapy, but with persistence of a fibrovascular RPE detachment, highlighting the pathomechanism of a classic CNV formation. Conclusions: Maculopathy in dengue fever may be followed by CNV as a result of the immunologic alteration of the RPE. Physicians should be aware of this manifestation to be able to initiate adequate treatment with excellent functional and anatomical results.
\end{abstract}

(C) 2015 S. Karger AG, Basel 
Veloso et al.: Choroidal Neovascularization Induced by Immunogenic Alteration of the Retinal Pigment Epithelium in Dengue Fever

\section{Introduction}

Dengue fever (DF), a tropical infection caused by the dengue virus, is a multisystemic disease characterized by fever, headache, muscle and joint pain [1]. The disease is transmitted by the bite of an infected Aedes aegypti mosquito. In cases with severe and repeated infection, hemorrhagic DF may develop due to exacerbation of the immune response. Ocular manifestations include intraretinal hemorrhages, retinal edema, cotton wool spots, uveitis, optic neuropathy and vascular sheathing [2]. Involvement of the foveal region has also been described [3, 4]. Optical coherence tomography (OCT) findings include 3 patterns of maculopathy: diffuse retinal thickening, cystoid macular edema and foveolitis [5]. To date, there have been no reports of choroidal neovascularization (CNV) secondary to DF. Herein, we report the initial manifestation of $\mathrm{CNV}$, the morphologic pathomechanism and the long-term follow-up of a case of CNV secondary to DF as well the successful response to treatment.

\section{Materials and Methods}

A 54-year-old female was referred to our department with blurred vision and metamorphopsia in her left eye following the diagnosis of DF 2 weeks earlier. She presented all classic systemic symptoms of DF with a papular rash over her arms and hands followed by fever, headache, retro-orbital pain and myalgia. A systemic workup revealed a thrombocytopenia, and a real-time PCR for the dengue virus type 2 was positive, confirming the diagnosis of DF. Noteworthy, she reported 2 previous episodes of DF in 2009 and 2011. Her best-corrected visual acuity (BCVA) was 20/20 in the right eye and 20/150 in the left eye. An anterior segment examination showed no abnormalities. Ophthalmoscopy revealed subretinal fluid and focal hemorrhage in the macula of the left eye (fig. 1a). A fluorescein angiography (FA) showed a hyperfluorescent lesion with well-defined borders and active leakage consistent with classic CNV (fig. 1b). A spectral domain OCT [SD-OCT; Spectralis OCT TM (Heidelberg Engineering, Heidelberg, Germany)] revealed subretinal fluid and focal hyperreflective material in the subretinal space as well as an area with small retinal pigment epithelium (RPE) detachments (fig. 1c). The patient was referred to receive 3 monthly intravitreal injections of ranibizumab (Lucentis; Novartis, Basel, Switzerland, and Genentech, Inc., South San Francisco, Calif., USA). After this loading dose, her BCVA improved to 20/25 in the left eye, and there also was a marked anatomical improvement (fig. 1d). After 6 months, her BCVA was still $20 / 25$ in the left eye and the SD-OCT remained without any signs of active CNV. Two years later, the patient again presented to our service complaining about a reduced VA in her left eye. The BCVA was 20/30 and the FA showed a leaking lesion temporal to and another site of leakage inferior to the fovea. Indocyanine green angiography revealed focal hot spots in these areas (fig. 2a, b). SD-OCT showed marked subretinal fluid, an increased extension of RPE detachments and RPE discontinuity, discrete intraretinal cysts and hyperreflective subretinal material (fig. 2c). The patient underwent 2 monthly intravitreal injections of ranibizumab with functional and anatomical improvement. The BCVA improved to 20/25, but there was persistence of fibrovascular RPE detachment (fig. 2d). Six months later, her VA had returned to 20/20 and OCT showed an absence of disease activity. Regular follow-up visits were recommended. 
Veloso et al.: Choroidal Neovascularization Induced by Immunogenic Alteration of the Retinal Pigment Epithelium in Dengue Fever

\section{Discussion}

$\mathrm{DF}$ is the most common mosquito-borne viral disease in humans. In recent years, it has become a major international public health concern. The disease is endemic in Brazil, but has emerged even in Europe, and the increasing prevalence has been accompanied by a rise in the proportion of severe cases [6]. Marked thrombocytopenia, for example, predisposes to severe forms of ocular disease [7, 8]. The mean interval between the systemic manifestations of DF and the onset of visual symptoms usually ranges from 6 to 7 days [2].

OCT is a noninvasive imaging technique that provides a better understanding of the pathogenesis, a more precise diagnosis and an improved monitoring of macular disorders. Teoh's classification of DF-related maculopathy into 3 groups is based on OCT patterns including retinal thickening in the central/paracentral fovea (type 1), cystoid macular edema with large cystoid-like spaces (type 2) and foveolitis with or without macular edema and high reflectivity at the level of the outer retina (type 3) [5]. All described changes are restricted to the neurosensory layers. Clinical, angiographic and tomographic findings of our case, however, are consistent with CNV, an entity associated with the alteration of the RPE layer. Secondary CNV always originates from retinochoroidal disease and is not only characterized by retinal features [9]. The precise pathogenesis of dengue maculopathy remains unknown. The immunopathologic mechanism probably comprises a complex series of immune responses initiated by primary infection with dengue virus, followed by antibody production. It is unknown whether autoantibodies in ocular disease may be directed against the retina or the RPE; nevertheless, alteration of the RPE barrier may consecutively induce CNV formation $[2,10,11]$. In age-related macular degeneration, the pathophysiologic role of the complement system has been highlighted impressively [12]. Complement activation leads to excessive damage to vascular and cellular barriers and potentially to RPE, preceeding CNV formation. Substantial RPE alteration was seen in our patient (including RPE discontinuity at the CNV ingrowth site and fibrovascular RPE detachments). Moreover, antibody-dependent enhancement occurs when non-neutralizing antiviral proteins facilitate virus entry into host cells, leading to increased infectivity in the cells. The phenomenon of antibody-dependent enhancement may be observed when a person who has previously been infected with one serotype of the dengue virus becomes infected many months or years later with a different serotype. In such cases, the clinical course of the disease is more severe and higher viremia occurs [13]. Our patient reported previous events of systemic disease. There is no specific treatment for systemic DF, and management revolves around appropriate supportive therapy and close monitoring. At present, there is also no measure to prevent ocular involvement during a dengue infection. Most ocular lesions, including retinal hemorrhages and foveolitis, resolve spontaneously over time. There is no known effective treatment for dengue maculopathy and there are no randomized trials to date. Active surveillance and corticosteroid therapy have been the main treatment modalities employed until now [14]. Systemic corticosteroids have been used in patients with maculopathy, uveitis, optic neuritis and neuromyelitis optica associated with DF and include oral prednisolone and/or intravenous methylprednisolone.

Generally, inflammatory changes often result in increased vascular endothelial growth factor (VEGF) levels [5]. VEGF is a diffusible cytokine that promotes angiogenesis and vascular permeability and it is considered an important factor promoting neovascularization. Ranibizumab is a recombinant, humanized monoclonal antibody that neutralizes all active forms of VEGF-A and is particularly effective in classic CNV, which is typically found in secondary CNV [15]. Herein, we report a case of CNV secondary to DF. To the best of our knowledge, this has not been previously described or treated. Considering the good treat- 
Veloso et al.: Choroidal Neovascularization Induced by Immunogenic Alteration of the Retinal Pigment Epithelium in Dengue Fever

ment response of $\mathrm{CNV}$ related to other diseases, we decided to treat our patient with intravitreal ranibizumab.

In conclusion, this is the first case to describe $\mathrm{CNV}$ as a complication of DF, highlighting the role of inflammatory mechanisms in the disease origin. SD-OCT is suggested as a useful diagnostic tool to differentiate neovascular disease from retinal inflammation alone, and intravitreal ranibizumab is suggested as an efficient therapeutic modality.

\section{Disclosure Statement}

None of the authors has proprietary interests or research funding.

\section{References}

1 Gubler DJ: The changing epidemiology of yellow fever and dengue, 1900 to 2003: full circle? Comp Immunol Microbiol Infect Dis 2004;27:319-330.

2 Lim WK, Mathur R, Koh A, Yeoh R, Chee SP: Ocular manifestations of dengue fever. Ophthalmology 2004;111:2057-2064.

3 Bacsal K, Chee SP, Cheng CL, Flores JVP: Dengue-associated maculopathy. Arch Ophthalmol 2007;125:501510.

4 Loh BK, Bacsal K, Chee S, Cheng BC, Wong D: Foveolitis associated with dengue fever: a case series. Ophthalmologica 2008;222:317-320.

5 Teoh SC, Chee CK, Laude A, Goh KY, Barkham T, Ang BS: Optical coherence tomography patterns as predictors of visual outcome in dengue-related maculopathy. Retina 2010;30:390-398.

6 Teixeira MG, Siqueira JB Jr, Ferreira GL, Bricks L, Joint G: Epidemiological trends of dengue disease in Brazil (2000-2010): a systematic literature search and analysis. PLoS Negl Trop Dis 2013;7:e2520.

7 Sumardi U, Nelwan EJ: Retinal hemorrhage in dengue hemorrhagic fever. Acta Med Indones 2011;43:66-67.

-8 Kumar V, Kataria R, Mehta V: Dengue hemorrhagic fever: a rare cause of pituitary tumor hemorrhage and reversible vision loss. Indian J Ophthalmol 2011;59:311-312.

-9 Rouvas A, Petrou P, Douvali M, Ntouraki A, Vergados I, Georgalas I, Markomichelakis N: Intravitreal ranibizumab for the treatment of inflammatory choroidal neovascularization. Retina 2011;31:871-879.

10 Leong AS, Wong KT, Leong TY, Tan PH, Wannakrairot P: The pathology of dengue haemorrhagic fever. Semin Diagn Pathol 2007;24:227-236.

-11 Lei HY, Yeh TM, Liu HS, Lin YS, Chen SH, Liu CC: Immunopathogenesis of dengue virus infection. J Biomed Sci 2001;8:377-388.

12 Camelo S: Potential sources and roles of adaptive immunity in age-related macular degeneration: shall we rename AMD into autoimmune macular disease? Autoimmune Dis 2014;2014:532487.

-13 Guzman MG, Alvarez M, Rodriguez-Roche R, Bernardo L, Montes T, Vazquez S, Morier L, Alvarez A, Gould EA, Kouri G, Halstead SB: Neutralizing antibodies after infection with dengue 1 virus. Emerg Infect Dis 2007;13:282-286.

Ng AW, Teoh SC: Dengue eye disease. Surv Ophthalmol DOI: 10.1016/j.survophthal.2014.07.003. Kaiser PK, Brown DM, Zhang K, Hudson HL, Holz FG, Shapiro H, Schneider S, Acharya NR: Ranibizumab for predominantly classic neovascular age-related macular degeneration: subgroup analysis of first-year ANCHOR results. Am J Ophthalmol 2007;144:850-857. 


\section{Case Reports in Ophthalmology}

\begin{tabular}{l|l}
\hline \multicolumn{2}{l}{ Case Rep Ophthalmol 2015;6:18-23 } \\
\hline DOI: 10.1159/000371791 & $\begin{array}{l}\text { @ 2015 S. Karger AG, Basel } \\
\text { www.karger.com/cop }\end{array}$ \\
\hline
\end{tabular}

Veloso et al.: Choroidal Neovascularization Induced by Immunogenic Alteration of the Retinal Pigment Epithelium in Dengue Fever
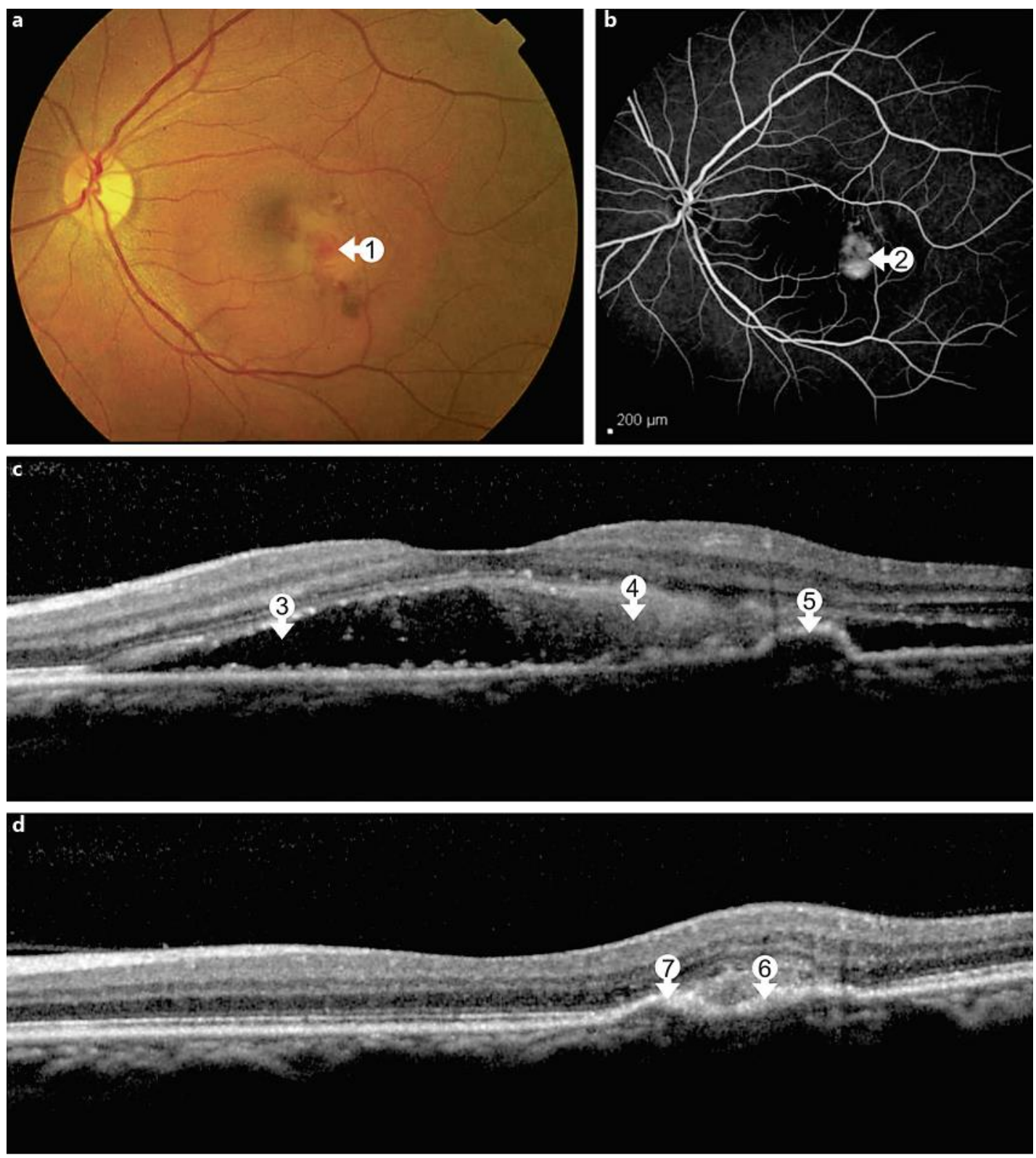

Fig. 1. a At baseline presentation, the macula of the left eye demonstrates focal hemorrhages and edema (arrow 1). b FA reveals active leakage from a small classic CNV lesion (arrow 2). c SD-OCT shows subretinal fluid (arrow 3), focal hyperreflective material above the retinal pigment epithelium (arrow 4) and RPE detachment (arrow 5). d Following the third intravitreal injection with ranibizumab, anatomical improvement with resolution of subretinal fluid is seen. In SD-OCT, a focal RPE scar (arrow 6) and a persistent RPE detachment (arrow 7) are seen. 
Case Reports in

Ophthalmology

\begin{tabular}{l|l}
\hline \multicolumn{2}{l|}{ Case Rep Ophthalmol 2015;6:18-23 } \\
\hline DOI: $10.1159 / 000371791$ & $\begin{array}{l}\text { C } 2015 \text { S. Karger AG, Basel } \\
\text { www.karger.com/cop }\end{array}$ \\
\hline
\end{tabular}

Veloso et al.: Choroidal Neovascularization Induced by Immunogenic Alteration of the Retinal Pigment Epithelium in Dengue Fever
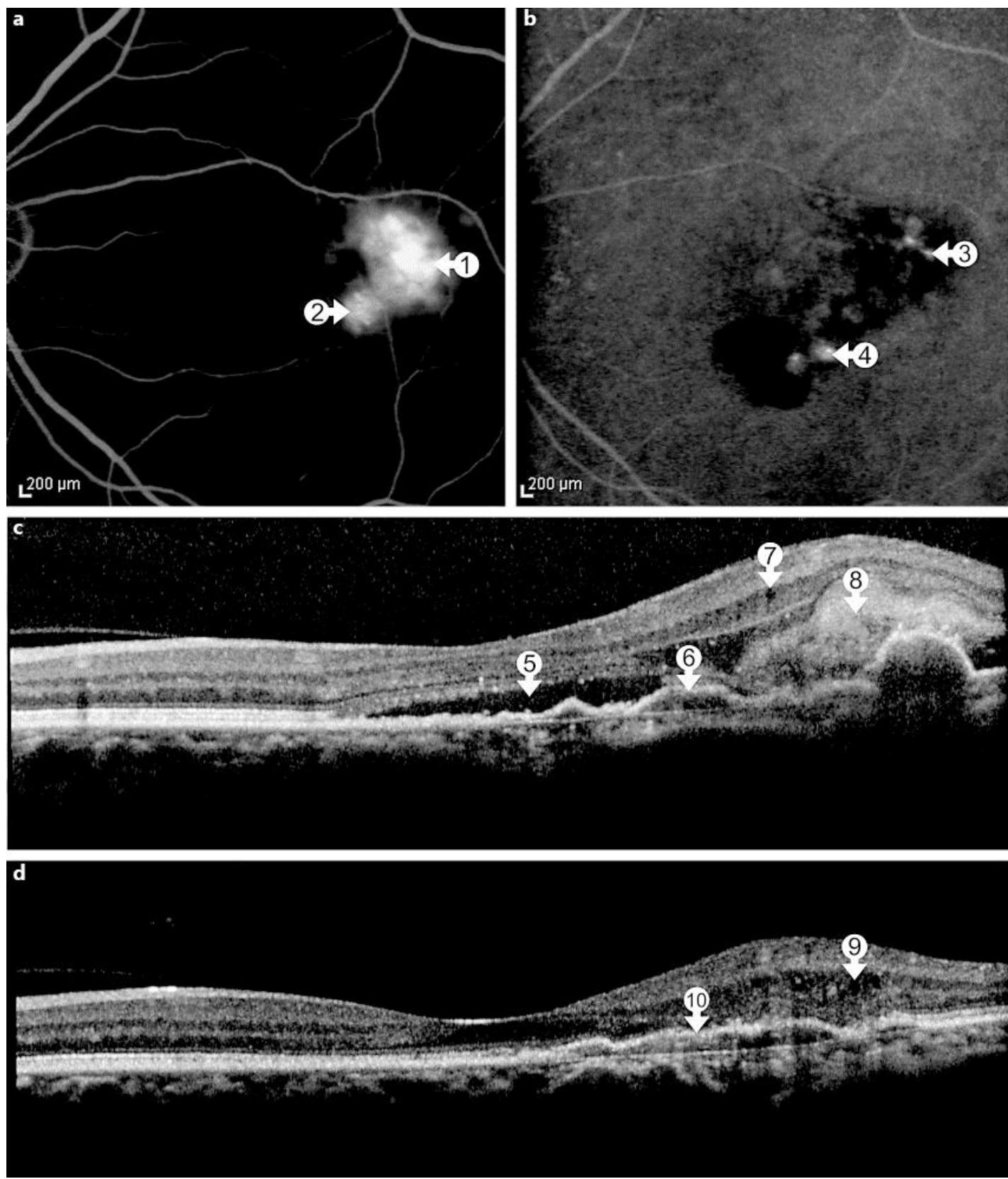

Fig. 2. a Two years later, FA identifies two active leakage sites temporal and inferior to the fovea (arrows 1 and 2). b Indocyanine green angiography reveals 2 separate hot spots, temporal and inferior to the fovea (arrows 3 and 4). c SD-OCT detects subretinal fluid (arrow 5) and an enlarged area with RPE irregularities and RPE detachment (arrow 6), intraretinal cysts (arrow 7) and hyperreflective subretinal material (arrow 8). d SD-OCT following successful treatment with ranibizumab injections shows persistent small intraretinal cysts (arrow 9) and a fibrovascular RPE detachment temporal to the fovea (arrow 10). 